\title{
HOMOGENEOUS LOCALLY COMPACT GROUPS WITH COMPACT BOUNDARY
}

\author{
BY \\ KARL HEINRICH HOFMANN (1)
}

A locally compact group with compact boundary is a locally compact topological semigroup in which an open subgroup is dense and has a compact complement. These semigroups were studied in a previous paper whose results and notation are freely used in the present work [2]. The general assumption made in this study in addition to the hypotheses mentioned is the existence of transitive group of homeomorphisms on the space of the semigroup. We restrict ourselves essentially to the connected case although the hypothesis we use actually is that the component of the identity of $G$ be not compact; the question of totally disconnected homogeneous groups with boundary is therefore unsettled.

The first main result which we obtain exhibits the fact that in a connected locally compact homogeneous group with boundary the influence of the boundary on the topological structure of $S$ is so strong that, if the boundary $B$ is finite dimensional, all of $S$ is finite dimensional. The method of the proof uses a local fibering of $S$ with cosets of the subsemigroup $K$ as fiber, where $K$ is the set of all elements $s$ such that $s e=e$ with the identity $e$ of $B$, and with neighborhoods of $B$ as bases. A group theoretical lemma which is of some interest in its own right has to be proved to obtain the theorem mentioned: If a locally compact group has a neighborhood of the identity whose homotopy groups relative to the identity vanish for all positive dimensions, then there are arbitrarily small neighborhoods which are topologically the product of an euclidean cell, a (not necessarily finite dimensional) solenoid and a totally disconnected compact subset of $G$; all small connected subgroups are solenoids. It is obvious what happens, then, if $G$ is in addition locally connected.

If the boundary $B$ of $G$ in $S$ is not only finite dimensional but also locally connected, then $S$ is a manifold, i.e. is locally euclidean; hence both $G$ and $B$ are Lie groups. Theorem II gives a full description of the structure of $S$ in this case. It is either a direct product of the full multiplicative semigroup of real numbers with some compact group or else the boundary is contained in an $L$ semigroup in the sense of Mostert and Shields whose results we use to dispose

Received by the editors July 21, 1961.

(1) This work was supported by the National Science Foundation. It has been accepted as part of the author's Habilitationsschrift by the Mathematisch-naturwissenschaftliche Fakultät der Universität Tübingen. 
of this case. Once we have got to the place where $S$ turns out to be a manifold, we are in a situation which was under more general circumstances studied by Hudson-Lester [4]; for $S$ is then a semigroup on an $n$-manifold with an $n-1$ dimensional compact subgroup $C$; we use, however, in our case essentially group theoretic methods and obtain specific information concerning the structure of the groups involved. As a corollary we get a theorem proved by the author [1], namely the fact that a locally compact connected homogeneous group with zero is isomorphic to either one of the multiplicative semigroups of the real, the complex or the quaternion field. In the more general case the fiber $K$ of all elements, for which the identity $e$ of $B$ is a zero has always this structure.

It is an open question whether the hypothesis of local connectedness of the boundary is actually essential in compiling the necessary information about the fiber $K$.

I. Homogeneous locally compact groups with compact finite dimensional boundary. The first definition is simply a repetition of a definition from [2]:

1.1. Definition. Let $S$ be a topological semigroup satisfying the following two conditions:

(i) $S$ is locally compact and satisfies the Hausdorff separation axiom.

(ii) $S$ contains an open dense subgroup $G$ such that the complement $B=S \backslash G$ is compact.

Then $G$ is called a locally compact group with compact boundary (l.c.g.c.b.), $G$ is called its maximal subgroup, $B$ is called the boundary [2].

We recall that $B$ is a compact topological group with identity $e$, that $x \rightarrow x e$ is an endomorphism of $G$ onto $B$ and that we denote the set of all $s$ in $S$ which satisfy $s e=e$ with $K$ and its intersection with the unique maximal compact subgroup $C$ of $G$ with $N$. The semigroup $K$ and the subgroup $N$ will play a very important rôle in this section. If $B=\{e\}$, then $S$ is called a locally compact group with zero (1.c.g.z.).

1.2. Definition. A 1.c.g.c.b. $S$ will be called homogeneous if the space $S$ has a transitive group of homeomorphisms, i.e. if for any pair, $x, y$, of points in $S$ there is a homeomorphism of $S$ mapping $x$ on $y$.

The following proposition will serve to restrict the possible structure of a l.c.g.c.b. considerably if we add the hypothesis of homogeneity.

1.3. Proposition Let $S$ be a homogeneous l.c.g.c.b. Then the following statements are equivalent:

(i) $G$ does not contain a compact open subgroup.

(ii) There is a connected subspace containing both 1 , the identity of $G$, and $e$ the identity of $B$.

(iii) $K$ is connected. 
(iv) There exists a subsemigroup $M_{0}$ isomorphic to the nonnegative reals under multiplication.

(v) $K$ is isomorphic to $\left(M_{0} \times N\right) / \rho^{\prime}$, where $\rho^{\prime}$ is the congruence relation which identifies all points of $\{0\} \times N$; i.e. $K$ is a l.c.g.z.

(vi) $S$ is isomorphic to $\left(M_{0} \times C\right) / \rho$, where $\rho$ is the congruence relation which collapses all cosets modulo $\{0\} \times N$ in $\{0\} \times C$.

(vii) There is an arc joining 1 and $e$.

Proof. Without the assumption of homogeneity we know that (i) and (ii) are equivalent (Theorem II [2]), and that (iii)-(vii) are equivalent (2.7 and Theorem IV [2]). Trivially (vii) implies (ii), so that we have to prove that homogeneity and (ii) imply (vii), for instance. If $S$ satisfies (ii), then there is a one-parameter subgroup $M$ in $G$ which is isomorphic to the positive reals under multiplication (Theorem II), and its closure in $S$ is not compact. Therefore the arc component of 1 cannot be relatively compact. By homogeneity, there exists a homeomorphism of $S$ mapping 1 onto $e$, and hence mapping the arc component of 1 onto the arc component of $e$. Thus the arc component of $e$ cannot be relatively compact in $S$, i.e. its closure is not compact. But then this arc component cannot be entirely contained in the compact subspace $B$. Therefore, there exists an element $g \in G$ and an arc joining $e$ with $g$. Since $x \rightarrow x g^{-1}$ is a continuous mapping of $S$ onto itself, and since $x \rightarrow x e$ is a continuous mapping of $S$ onto $B$, we know that $e g^{-1}$ and $g g^{-1}=1$ on one hand and $e$ and $e g$ on the other hand are joined by an arc. But the last connection again implies that $e g^{-1}$ and $(e g) g^{-1}=e 1=e$ are joined by an arc. Hence $e$ and 1 are joined by an arc which finishes the proof.

From now on we confine our attention exclusively to such 1.c.g.c.b. which satisfy any one of the conditions of Proposition 1.3. Also, $\rho$ will continuously denote the congruence relation on $M_{0} \times C$ collapsing the cosets modulo $\{0\} \times N$ in $\{0\} \times C$.

1.4. Proposition. Let $S$ be a l.c.g.c.b. isomorphic to $\left(M_{0} \times C\right) / \rho$. Then the connected component $S_{e}$ of $e$ is isomorphic to $\left(M_{0} \times C^{\prime}\right) / \rho$, where $C^{\prime}=C_{1} N$ with the connected component $C_{1}$ of 1 in $C$.

Proof. (a) $\left(M_{0} \times C^{\prime}\right) / \rho$ is connected; for $\left(\{0\} \times C^{\prime}\right) / \rho$ is isomorphic to $C_{1} N / N$ which is a homeomorphic image of the connected group $C_{1}$. Any point $\rho(m, c)$ with $m \neq 0, c \in C^{\prime}$ is contained in the connected subspace $\rho\left(M_{0} \times\{c\}\right)$ intersecting $\left(\{0\} \times C^{\prime}\right) / \rho=\rho\left(\{0\} \times C^{\prime}\right)$. (b) Let $x$ be an element not contained in $\left(M_{0} \times C^{\prime}\right) / \rho$. Then $x=\rho(m, c)$, and $c$ is not in $C^{\prime}$. Hence $\rho(0, c)$ is not in $\left(\{0\} \times C^{\prime}\right) / \rho$. We shall prove that then there is a compact open subgroup $C^{\prime \prime}$ of $C$ such that the compact open subgroup $\left(\{0\} \times C^{\prime \prime}\right) / \rho$ does not contain $\rho(0, c)$; this holds, for $C / C^{\prime}$ is totally disconnected and compact because $C^{\prime}$ contains the component $C_{1}$ and because $C$ is compact; but then, given any element in $C / C^{\prime}$ other than the identity, one finds a compact open suboroup in $C / C^{\prime}$ not con- 
taining this element $[6$, p. 54,56$]$. The group $(C / N) /\left(C^{\prime} / N\right)$ is isomorphic to $C / C^{\prime}$, and $(\{0\} \times C) /\left(\{0\} \times C^{\prime}\right)$ is isomorphic to $C / C^{\prime}$. Hence there is indeed a normal compact open subgroup $C^{\prime \prime}$ of $C$ such that $\left(\{0\} \times C^{\prime \prime}\right) /\left(\{0\} \times C^{\prime}\right)$ does not contain the coset of $(0, c)$ modulo $\left(\{0\} \times C^{\prime}\right)$. If $\rho(0, c)$ is not in $\left(\{0\} \times C^{\prime \prime}\right) / \rho$ then $\rho(m, c)$ is not in $\left(M_{0} \times C^{\prime \prime}\right) / \rho$; but $\left(M_{0} \times C^{\prime \prime}\right) / \rho$ is open and closed in $\left(M_{0} \times C\right) / \rho$; for it is closed since $\rho$ is closed, and if $C^{+}$is the complement of $C^{\prime \prime}$ in $C$, then $M_{0} \times C^{+}$is closed and, therefore $\left(M_{0} \times C^{+}\right) / \rho$ is closed since $\rho$ is closed. But $\left(M_{0} \times C\right) / \rho$ is the disjoint union of $\left(M_{0} \times C^{\prime \prime}\right) / \rho$ and $\left(M_{0} \times C^{+}\right) / \rho$. This finishes the argument.

1.5. Definition. Let $S$ be isomorphic to $\left(M_{0} \times C\right) / \rho$. The endomorphism $x \rightarrow x e$ of $S$ onto $B$ will be denoted by $\pi$. The 1.c.g.c.b. $S$ is said to admit a local cross section over $b_{0} \in B$ if there exists a neighborhood $U$ in $B$ of the point $b_{0}$ and a continuous mapping $\phi$ of $U$ into $G$ such that $\pi \circ \phi$ is the identity map of $U$. Thus, whenever $\phi$ defines such a local cross section, we have $\phi(b) e=b$ for all $b \in U$.

1.6. Proposition. Let $S$ be a l.c.g.c.b. isomorphic to $\left(M_{0} \times C\right) / \rho$. If $C$ has a local cross section for $N$, then $S$ admits a local cross section over every $b \in B$.

Proof. Suppose that $C$ has a local cross section for $N$, i.e. there is a neighborhood $U^{\prime}$ of each point in $C / N$ and a continuous mapping $\phi^{\prime}$ of $U^{\prime}$ into $C$ such that this mapping followed by the quotient mapping $\pi^{\prime}$ of $C$ onto $C / N$ is the identity on $U^{\prime}$. Let $\psi$ be the obvious isomorphism of $C / N$ onto $B$. Let $U=\psi\left(U^{\prime}\right)$ and $\phi=\phi^{\prime} \circ \psi^{-1}$. Since $\pi=\psi \circ \pi^{\prime}$, we have indeed $\pi \circ \phi=\psi \circ \pi^{\prime}$ $\circ \phi^{\prime} \circ \psi^{-1}=$ identity on $U$.

1.7. Proposition. If $S$ is a l.c.g.c.b. isomorphic to $\left(M_{0} \times C\right) / \rho$, and if $C / N$ is finite dimensional, then $S$ admits a local cross section over every $b \in B$.

Proof. A theorem of Mostert [7, p. 65] shows that the hypotheses of 1.6 are satisfied.

1.8. Proposition. Let $S$ be a l.c.g.c.b. isomorphic to $\left(M_{0} \times C\right) / \rho$. Suppose that $S$ admits a local cross section over $b \in B$. Let $\phi$ be the mapping of a neighborhood $U$ of $b_{0}$ in $B$ into $G$ such that $\pi \circ \phi$ is the identity on $U$. Then the mapping $(k, b) \rightarrow k \phi(b)$ is a homeomorphism of $K \times U$ onto $S$.

Proof. The mapping $\chi:(k, b) \rightarrow k \phi(b)$ is clearly continuous, and so is the mapping $\chi^{\prime}: s \rightarrow\left(s \phi(s e)^{-1}, s e\right)$ of $\phi^{-1}(U)$ into $K \times U$; since $\pi\left(s \phi(s e)^{-1}=s e(s e)^{-1}\right.$ $=e$, this mapping goes indeed into $K \times U$. We show that the mappings $\chi$ and $\chi^{\prime}$ are inverses of each other which will prove the assertion. $\chi^{\prime}(\chi(k, b))=\chi^{\prime}(k \phi(b))$ $\left(k \phi(b) \phi(k \phi(b) e)^{-1}, k \phi(b) e\right)=\left(k \phi(b) \phi(b)^{-1}, b\right)=(k, b)$ since $k \phi(b) e=(k e) \phi(b) e$ $=e b=b$ by the definition of $K$ and the characteristic property of $\phi$ (see 1.5.). Conversely, $\chi\left(\chi^{\prime}(s)\right)=\chi\left(s \phi(s e)^{-1}, s e\right)=s \phi(s e)^{-1} \phi(s e)=s$. 
1.9. Proposition. Let $S$ be a l.c.g.c.b. in which there is an arc joining $e$ and 1 . Suppose that $B$ is n-dimensional. Then e has arbitrarily small neighborhoods $V$ in $B$, such that there is an $n$-cell $E$ in $B$ containing $e$, and a totally disconnected compact subgroup $T$ of $B$ such that $V=E T$ and the mapping $(k, x, t)$ $\rightarrow k \phi(x t)$ is a homeomorphism of $K \times E \times T$ onto $K \phi(V)$ where $\phi$ is a mapping from $V$ into $G$ such that $\pi \circ \phi$ is the identity on $V$; such a mapping exists for sufficiently small $V$ by 1.7 .

Proof. It is sufficient to remember that there are sufficiently small neighborhoods of the identity $e$ in the compact $n$-dimensional group $B$ which are topologically (and, by the way, algebraically) direct products of some $n$-cell $E$ and a totally disconnected subgroup T. (See e.g. [10, p. 93].) Then Proposition 1.8 proves the rest.

1.10. Proposition. Let $S$ satisfy the conditions of Proposition 1.9 and the additional condition that $S$ is homogeneous. Then every point $s$ in $S$ has arbitrarily small neighborhoods $U$ such that $\pi_{n}(U, s)=\{0\}$ for $n=1,2, \ldots$, i.e. $S$ is locally homotopically trivial. Moreover, the neighborhoods $U$ are homeomorphic to direct products of $U_{0}$ and $T$, where $U_{0}$ is a subset of $U$ homotopically contractible to $s$ and $T$ is totally disconnected. Furthermore, $U_{0}$ is first countable at $s$.

Proof. Because of homogeneity it is sufficient to prove the proposition for $s=e$. By 1.9, $e$ has neighborhoods $K \phi(V)$ homeomorphic to $K \times E \times T$ (same notation as in 1.9). Now $K$ is a 1.c.g.z. (1.3.v) and the set $\left(S_{0} \cup S_{1}\right) \cap K$ of all of its points whose powers either converge to $e$ or form a relatively compact set (see $[2,1.10])$ is a neighborhood of $e$ and a cone with vertex $e$ and compact base $K \cap C$. Let $k_{m}$ be a sequence of elements in $K \backslash\{e\}$ converging to $e$. Then the sets $W_{m}=k_{m}\left(S_{0} \cup S_{1}\right) \cap K$ form a neighborhood base for $e$ in $K$ [2], 1.4. Since all mappings $x \rightarrow k_{m} x$ of $S$ onto itself are homeomorphisms, all $W_{m}$ are cones with vertex $e$ and compact base. Hence $\pi_{n}\left(W_{m} e\right)=\{0\}$ for $n=1,2, \ldots$ and all $m$. The point $e$ has arbitrarily small neighborhoods of the form $W_{m} \phi E T$ homeomorphic to $W_{n} \times E \times T$. Then $\pi_{n}\left(W_{m} \phi(E T)\right)$ is isomorphic to $\pi_{n}\left(W_{n}\right)$ $+\pi_{n}(E)+\pi_{n}(T)=0+0+0$ for $n=1, \ldots$, (see [3, p. 144]). If $U_{0}=W_{n} \phi(E)$, then clearly $U_{0}$ is homotopically contractible to $e$, and $U$ is homeomorphic to $U_{0} \times T$. Moreover, $U_{0}$ is first countable at $e$, since $W$ is first countable.

1.11. LeMMA. Let $C$ be a compact connected group and $L$ a closed Lie group in $C$ satisfying one or the other of the following conditions:

(i) $L$ is simple,

(ii) $L$ is one-dimensional and normal in $C$.

Then there is a homomorphism $f$ of $C$ onto a Lie group $L^{\prime}$ such that the restriction $f^{\prime}$ of $f$ to $L$ is a covering homomorphism, i.e. a local isomorphism. 
Proof. Suppose (i) is satisfied. Because of the well-known structure of compact connected groups we assume that there is a connected compact abelian group $A$, a simple simply connected Lie group $L_{0}$, a direct product $P$ of simple simply connected Lie groups, and a totally disconnected subgroup $D$ of the center of $A \times P \times L_{0}$ such that

(a) $C$ is equal to $\left(A \times P \times L_{0}\right) / D$,

(b) $L$ is equal to $\left(\{1\} \times\{1\} \times L_{0}\right) D / D$.

(See $[10$, pp. 88-93].)

Let $F=(A \times P \times\{1\}) D / D, L^{\prime}=C / F$ and $f$ the quotient projection. Then the image of $L$ is isomorphic to $L /(L \cap F)$ and is obviously all of $L^{\prime}$. Since all elements of $(A \times P \times\{1\}) D$ commute with all elements of $\left(\{1\} \times\{1\} \times L_{0}\right)$, the group $F$ is in the centralizer of $L$, therefore the intersection $L \cap F$ is contained in the center of the simple group $L$ which is finite. Hence $L$ is a covering group of $L /(L \cap F)$ and, therefore, of $L^{\prime}$.

Suppose (ii) is satisfied. $C$ contains a central compact connected subgroup $Z$ and a normal subgroup $Y$ which is a projective limit of semisimple Lie groups, and $Z=Z Y$ and $Z \cap Y$ is totally disconnected [10, p. 90]. $L$ is contained in $Z$, because it is normal and hence central. But then it is a direct factor (see e.g. $\left[9\right.$, p. 52]), i.e. $Z=L Z^{\prime}, L \cap Z^{\prime}=1$. Let now $F=Z^{\prime} Y, L^{\prime}=C / F$ and $f$ the quotient projection. The image of $L$ is all of $L^{\prime}$ and is isomorphic to $L /(L \cap F)$. If we can show that $L \cap F$ is not all of $L$, the proof is accomplished, because any proper closed subgroup of the circle group $L$ is finite. If, however, $L$ were contained in $F=Z^{\prime} Y$, then the circle group $L Z^{\prime} / Z^{\prime}$ would be contained in the center of $Z^{\prime} Y / Z^{\prime}$ which is a projective limit of semisimple Lie groups and has, therefore, a totally disconnected center.

1.12. Lemma. Let $f_{1}: L \rightarrow L_{1}$ be a covering homomorphism of the simply connected compact $n$-dimensional group $L$ onto $L_{1}$ and let $f_{2}: L_{1} \rightarrow L_{2}$ be a covering homomorphism. Then

$$
f_{1}^{*}: \pi_{n}(L, 1) \rightarrow \pi_{n}\left(L_{1}, 1_{1}\right)
$$

and

$$
f_{2}^{*}: \pi_{n}\left(L_{1}, 1_{1}\right) \rightarrow \pi_{n}\left(L_{2}, 1_{2}\right)
$$

are isomorphisms and $\pi_{n}(L, 1)$ is isomorphic to $H_{n}(L)$.

Proof. The dimension of a simply connected compact group is at least 3 . The first two isomorphisms are a well-known property of covering spaces [3, Proposition 7.1, p. 154]. The last isomorphism is established by Hurewicz's theorem [3, Theorem 44, p. 148].

1.13. LEMMA. Let $f: L \rightarrow L_{1}$ be a covering homomorphism of a circle group $L$. Then $f^{*}: \pi_{1}(L, 1) \rightarrow \pi_{1}\left(L_{1}, 1_{1}\right)$ is a monomorphism.

Proof. See e.g. [3, Proposition 7.1, p. 154]. 
1.14. LemMa. Let $C$ be a compact connected group, $L$ a simple subgroup or a normal circle group. Let $n$ be the dimension of L. Suppose that $X$ is a subset of $C$ containing $L$. Then.

$$
\pi_{n}(X, 1) \neq 0 .
$$

Proof. Consider the following diagram:

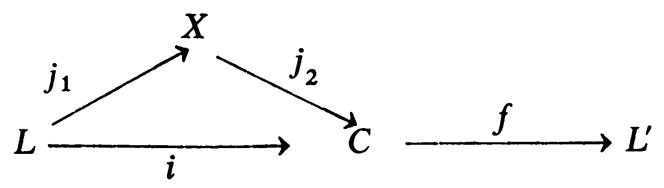

where $i, j_{1}, j_{2}$ are inclusion maps and $f: C \rightarrow L^{\prime}$ is as in Lemma 1.11; to this diagram there corresponds the following diagram involving the $n$th homotopy groups of the spaces:

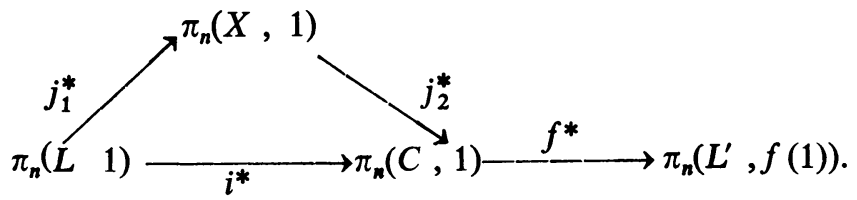

Now, if $=f^{\prime}$ is, according to Lemma 1.11, a covering homomorphism. Hence, by Lemmas 1.12 and $1.13, i^{*} f^{*}$ is an isomorphism if $n>1$ and a monomorphism if $n=1$. The group $\pi_{n}(L, 1)$ is isomorphic to an infinite cyclic group, if $n=1$ and to $H_{n}(L) \neq 0$, where $L$ is the simply connected covering group of $L(1.12)$. Hence

$$
f^{*} j_{2}^{*} j_{1}^{*}=f^{*} i^{*}
$$

is not trivial; therefore $j_{1}^{*}$ is not trivial, which implies

$$
\pi_{n}(X, 1) \neq 0 \text {. }
$$

1.15. Lemma. Let $G$ be a locally compact group such that the identity has a neighborhood $U$ satisfying

$$
\pi_{n}(U, 1)=0, \quad i=1,2, \ldots .
$$

Then every normal compact connected subgroup contained in $U$ is a solenoid.

Proof. Since every connected subgroup of $G$ is contained in the component $G_{0}$ of 1 and since $U \cap G_{0}$ is a neighborhood of 1 in $G_{0}$ having trivial homotopy groups of positive dimension we may assume that $G$ is connected. By Iwasawa's theorem [5, p. 549], $G$ is topologically the product of a vector space and a maximal connected compact subgroup of $G$; hence we may assume that $G$ is also compact.

Let $C$ be a compact connected subgroup of $G$ in $U$. Then $C$ is the product of a central connected abelian group $Z$ and a projective limit $Y$ of semisimple groups $[10$, p. 90$]$. If $Y$ were not trivial, then $C$ would contain at least one simple sub- 
group $[10$, p. 91$]$. Hence $C$ is abelian; it contains no torus subgroup of any dimension by 1.14 . This, however, is the type of group which we call a solenoid, since every one-parameter group is a one-to-one continuous image of the real line.

The reader may observe that in the case that $G$ is connected, $C$ is, as a connected compact abelian normal subgroup in the center of $G$. If we had required only $\pi_{n}(U, 1)=0, i=2,3, \ldots$, then the conclusion would be that every normal compact subgroup in $U$ is abelian.

1.16. MAIN LEMMA. Let $G$ be a locally compact group. If the identity has a neighborhood $U$ such that

$$
\pi_{n}(U, 1)=0, \quad n=1, \ldots,
$$

then $G$ has arbitrarily small neighborhoods $V$ such that there is a euclidean cell $E$ and a solenoid $Z$ (i.e. a compact abelian group in which every one-parameter subgroup is a one-to-one continuous image of the reals) and a totally disconnected compact subspace $T$, and $V=E Z T$, and this product is topologically direct.

Proof. $G$ contains an open subgroup which is a projective limit of Lie groups. Hence we may assume that $G$ is itself a projective limit of Lie groups. Let $W$ be a given neighborhood of the identity. Let $H$ be a normal compact subgroup of $G$ such that $H \subset U \cap W$ and $G / H$ is a Lie group. Since $H$ admits a local cross section (see [7, p. 65]), there is a euclidean cell $E$ containing 1 in $G$ such that $E H$ is topologically a direct product and a neighborhood of 1 . Now $H$ is a compact group. Let $H_{0}$ be its (characteristic) connected component. Then by 1.15, $H_{0}$ is a solenoid. $H_{0}$ admits a local cross section in $H$; hence there is a totally disconnected compact subspace $T$ containing 1 such that $H_{0} T$ is topologically a direct product and a neighborhood of 1 in $H$. The neighborhood $V=E H_{0} T$ is contained in $W$ and satisfies the requirements of the lemma.

1.17. Proposition. Let $S$ be a homogeneous l.c.g.c.b. such that an arc joins 1 and $e$. Suppose that $B$ is finite dimensional. Then $S$ is finite dimensional.

Proof. By Lemma 1.10, each point of $S$ has arbitrarily small neighborhoods all of whose homotopy groups of positive dimension vanish. Hence, by the Main Lemma 1.16, the identity 1 has a neighborhood of the form EZT, where $E$ is a euclidean cell, $Z$ is a solenoid, $T$ is a zero dimensional space, and the product is topologically direct. We shall show that $Z$ is finite dimensional; then $E Z T$ is finite dimensional [7], and the proof will be finished.

Let $U_{0} T^{\prime}$ be a neighborhood of 1 contained in EZT such that $U_{0}$ is homotopically contractible to 1 (hence arcwise connected) and $T^{\prime}$ is some zero dimensional space (1.10). Then $U_{0}$ is in EZ, the component of 1 in EZT. Let $p: E Z \rightarrow Z$ be the projection of the product $E Z$ on the factor $Z$. Then $A=p\left(U_{0}\right)$ is arcwise connected in $Z$. 
We shall show that $p\left(U_{0}\right)$ cannot contain a full one-parameter group of $Z$. Then, a fortiori, $U_{0}$ cannot contain a one-parameter group of $Z$, because such would have to be in $U_{0} \cap Z$ and then in $p\left(U_{0}\right)$. Thus $U_{0} T^{\prime}$ cannot contain a one-parameter group of $Z$, and $V=U_{0} T^{\prime} \cap Z$ is a neighborhood of 1 in $Z$ which cannot contain a connected subgroup. If, now, $H$ is a subgroup of $Z$ in $V$ such that $Z / H$ is a Lie group, then $H$ is totally disconnected and $Z$ is finite dimensional $[7$, p. 67]. It is, therefore, left to prove that a compact arcwise connected subset $A$ in a solenoid $Z$ cannot contain a one-parameter group.

Let $f: R \rightarrow Z$ be a continuous one-to-one representation of the reals $R$ into $Z$ such that the image and, consequently, also its closure $F$ is in $A$. Then every point of $F$ lies on a one-parameter group in $Z$ because the arc component in a compact group is covered by one-parameter groups [0]. But all these are torsion free so that $F$ is torsion free and connected. Thus its character group is divisible and torsion free [9] and is therefore a rational vector space. Since its character group is a direct sum of one-dimensional rational groups, $F$ is a direct product of duals of one-dimensional rational vector groups. We may from now on assume that $F$ is one-dimensional and that its character group $Q$ is a rational vector group. $F$ is itself not arcwise connected, hence there are points in $F$ which are contained in a one-parameter group contained in $Z$ but not in $F$; let $g: R \rightarrow Z$ be a representation of $R$ into $Z$ defining such a one-parameter group. Then there is a smallest positive real number $r$ such that $g(r) \in F$ and $Y=g(R) F$ $=g([0, r]) F$ is a compact connected group. Since $s \rightarrow g(s) F$ is one-to-one from $[0, r)$ onto $Y / F$, it is clear that $Y / F$ is a circle group. Hence $Y$ is an extension of $F$ by a circle group; thus its character group $Y^{\prime}$ is the extension of a cyclic infinite group $P$ by the rational group $Q$.

On the other hand, $Y=g([0, r]) F$ is certainly arcwise connected in Z. Hence, all the points of $Y$ lie on one-parameter groups in $Z$, all of which are torsion free. Hence $Y$ is torsion free which implies that $Y^{\prime}$ is divisible (it is torsion free since $Y$ is connected). Hence $Y^{\prime}$ is a rational vector group and therefore cannot be an extension of an infinite cyclic group by a rational vector group, since any divisible extension of a cyclic group has torsion elements in the factor group. This contradiction finally proves the proposition.

1.18. Proposition. Let $S$ be as in 1.17, but suppose in addition that $B$ is locally connected. Then $B$ and $G$ are Lie groups and $S$ is a manifold.

Proof. The compact finite dimensional locally connected group $B$ is a Lie group [10, p. 93]. Hence, by $1.10, S$ is locally arcwise connected since the totally disconnected factor disappears. But a finite dimensional locally arcwise connected group is a Lie group [6, p. 185]. Hence $G$ is a Lie group and $S$ is locally euclidean.

We collect the information gathered so far in the following: 
THEOREM I. Let $S$ be a homogeneous locally compact group with compact boundary in which there is an arc joining the identity 1 and the identity $e$ of the boundary $B$ (or, equivalently, in which the maximal subgroup $G$ aoes not contain a compact open subgroup). Then $S$ is finite dimensional if and only if the boundary $B$ is finite dimensional. $S$ is a manifold and both $G$ and $B$ are Lie groups if and only if the boundary $B$ is finite dimensional and locally connected.

II. Homogeneous locally compact groups with compact finite dimensional and locally connected boundary. In this section $S$ will always be a homogeneous 1.c.g.c.b. in which the boundary is finite dimensional and locally connected. Since the structure of $S$, as seen from our standpoint, is completely reflected by the connected component of $e$ we assume until the end that $S$ is connected. This implies that $C=C_{1} N$ with the connected component $C_{1}$ of the identity in $C$.

2.1. Proposition. Let $S$ be a connected l.c.g.c.b., and let the boundary be finite dimensional. Suppose in addition that $C=C_{1}$ is connected. Then $C$ is isomorphic to the quotient of a direct product of a Lie group $L$ and the group $N$ which is a sphere group of dimension 0,1 , or 3 , modulo a discrete cyclic group $D$ whose order in the case of $\operatorname{dim} N=0,3$ is at most 2.

Proof. $S$ is isomorphic to $\left(M_{0} \times C\right) / \rho$ as indicated in 1.3. vi. Let $H_{0}$ be the subsemigroup of $M_{0}$ which is isomorphic to the interval [0,1] under multiplication. Then $\left(H_{0} \times C\right) / \tilde{\rho}$, where $\tilde{\rho}$ is the congruence relation induced on $H_{0} \times C$ by $\rho$, is a $L$-semigroup in the sense of Mostert and Shields. Then $N$ is a sphere $[8$, p. 139]. It remains to determine the structure of $C$. As a compact connected Lie group $C$ is the product of a central torus $A$ and a semisimple group. Suppose first that $N$ is the three sphere. Then the semisimple part of $C$ is a product of a semisimple group $L_{0}$ and $N$; we set $L=A L_{0}$. Then all elements of $L$ commute with all elements of $N$. Hence, the intersection of $L$ and $N$ is in the center of $N$ which consists of two elements. Hence, by [10, p. 21 and p. 158] $C$ is isomorphic to the quotient of $L \times N$ modulo a subgroup isomorphic to $L \cap N$. Let now $N$ be a one-sphere. Then $A$ is the direct product of $N$ and a supplementary torus $A_{0}$. Let now $L_{0}$ be the full semisimple part of $C$ and put $L=A_{0} L_{0}$. Then by the same reason as before $C$ is isomorphic to the quotient of $L \times N$ modulo a subgroup isomorphic to $N \cap L$. Since $N$ is certainly not contained in $L$, this intersection is a closed subgroup of the circle group and is therefore cyclic. No statements can be made about the order in this case, since there are simple Lie groups whose centers are cyclic groups of any order. The case $\operatorname{dim} N=0$ is apparently trivial with $L=C$.

REMARK. The conditions given in Proposition 2.1 are apparently necessary and sufficient, since $S$ is a connected manifold, if they are satisfied. 
2.2. Proposition. Let $S$ be as in Proposition 2.1 but assume this time that $C$ is not connected, i.e. $C=C_{1} N$ with the component $C_{1}$ of $C$. Then $N$ is a 0 sphere (i.e. a two-element group) and $S$ is isomorphic to the direct product of $C_{1}$ and the full semigroup of real numbers under multiplication. (See also $[4$, p. 14].)

Proof. $G=S \backslash B$ is not connected and has as many components as the finite index of $C_{1}$ in $C$ indicates. Clearly $\operatorname{dim} S=\operatorname{dim} C+1$. Since the submanifold $B$ separates the manifold $S$, we have $\operatorname{dim} S=\operatorname{dim} B+1$; therefore $\operatorname{dim} C=\operatorname{dim} B$ $=\operatorname{dim} C / N=\operatorname{dim} C-\operatorname{dim} N$; hence $\operatorname{dim} N=0$, i.e. $N$ is discrete. But an $n-1$ dimensional orbit can separate an $n$-manifold in at most two parts (since "it has only two sides'). Hence the order of $N$ is two. The product $C_{1} N$ is therefore direct. Let $R=M_{0} N$; then $R$ is obviously isomorphic to the full semigroup of real numbers. The mapping $(r, c) \rightarrow r c$ of $R \times C_{1}$ onto $R C_{1}=S$ is one-to-one continuous. It is open on $(R \backslash\{e\}) \times C_{1}$ and is homeomorphic on every compact neighborhood of $\{e\} \times C_{1}$; hence it is a homeomorphism. It is clearly an algebraic isomorphism, since $R$ is central.

REMARK. If $S$ is no longer connected, then $G=S \backslash B$ is still the direct product of $C$, the maxima compact subgroup and $M_{0} \backslash\{e\}$, a one-parameter group [2], however $S$ need no longer be the direct product of $R=M_{0} N$ and some compact subgroup $C^{\prime}$ of index two in $C$, since there are enough compact totally disconnected groups containing a normal subgroup $N$ with two elements, which does not split in $C$.

We summarize our results in the following:

THEOREM II. Let $S$ be a locally compact group with compact boundary whose boundary is finite dimensional and locally connected. Let $C$ be the maximal compact subgroup containing the identity 1 of $S, B$ the boundary of the maximal subgroup $G$ with the identity e. Let $K=\{s: e s=s\}$, the kernel of the Clifford-Miller endomorphism, and $N=C \cap K$. (See [2, Theorem II].) Suppose that 1 and e are contained in some connected subspace. Then the following conditions are necessary and sufficient that $S$ be homogeneous:

(A) $S$ contains a subsemigroup $M_{0}$ isomorphic to the multiplicative semigroup of nonnegative reals, $C$ is a Lie group, and $S$ is isomorphic to $\left(M_{0} \times C\right) / \rho$ where $\rho$ is the congruence relation which identifies in the group $\{e\} \times C$ all cosets modulo the normal subgroup $\{e\} \times N$.

(B) $N$ is a sphere group, i.e. a group of two elements, a circle group or the group of quaternions with unit norm and $K$ is isomorphic to the full multiplicative semigroup of the reals, the complexes or the quaternions.

(C) Either one of the following cases occurs:

(CA) The maximal compact subgroup $C \cap S^{\prime}$ of the connected component $S^{\prime}$ of 1 is not connected, then $S^{\prime}$ is isomorphic to the direct product of $C \cap S^{\prime}$ and the full semigroup of reals; 
(CB) $C \cap S^{\prime}$ is connected and is isomorphic to the quotient of the direct product of a compact connected Lie group $L$ and $N$ modulo a finite cyclic group of the center of this product whose order is at most two in the cases where $\operatorname{dim} N=0,3$.

If $B$ contains only the point $e$, then $S=K$ and we have the following

COROLLARY II.1. A homogeneous locally compact connected group with zero is isomorphic to the full multiplicative semigroup of the reals, the complexes or the quaternions [1].

\section{REFERENCES}

0. M. J. Dixmier, Quelques propriétés des groups abéliens localement compacts, Bull. Sci. Math. 81 (1957), 38-48.

1. K. H. Hofmann, Lokalkompakte zusammenhängende topologische Halbgruppen mit dichter Untergruppe (und Erratum), Math. Ann. 140 (1960), 22-32 (resp. Math. Ann. 140 (1960), 442).

2. - Locally compact semigroups in which a subgroup with compact complement is dense, Trans. Amer. Math. 106 (1963), 19-51.

3. S. T. Hu, Homotopy theory, Academic Press, New York, 1959.

4. A. Hudson-Lester, On the structure of semigroups with identity on a noncompact manifold, Michigan Math. J. 8 (1961), 11-19.

5. K. Iwasawa, On some types of topological groups, Ann. of Math. (2) 50 (1949), 507-557.

6. D. Montgomery and L. Zippin, Topological transformation groups, Interscience, New York, 1955.

7. P. S. Mostert, Sections in principal fibre spaces, Duke Math. J. 23 (1956), 57-72.

8. P. S. Mostert and A. Shields, On the structure of semigroups on a compact manifold with boundary, Ann. of Math. 65 (1957), 117-143.

9. L. S. Pontryagin, Topologische Gruppen. II, German translation: Teubner, Leipzig, 1957.

10. A. Weil, L'intégration dans les groupes topologiques et ses applications, Actualités Sci. Ind. No. 1145 , Hermann, Paris, $1951=$ No. $869,1940$.

TULANE UNIVERSITY,

New OrLeans, Louisiana

UNIVERSTTÄT TÜBINGEN,

TÜBINGEN, GERMANY 